\title{
CCD survey with the CMASF from the Southern Hemisphere
}

\author{
M. Vallejo ${ }^{1}$, J. L. Muiños ${ }^{1}$, F. Belizón ${ }^{1}$, F. J. Montojo ${ }^{1}$ \\ C. C. Mallamaci ${ }^{2}$, J. A. Pérez ${ }^{2}$ L. F. Marmolejo ${ }^{2}$, \\ J. L. Navarro ${ }^{2}$, J. Sedeño ${ }^{2}$ \\ ${ }^{1}$ Real Instituto y Observatorio de la Armada, San Fernando, Spain. email: ppmu@roa.es \\ ${ }^{2}$ Observatorio Astronómico Félix Aguilar, San Juan, Argentina. email: ccmalla@gmail.com
}

\begin{abstract}
A subcatalogue with positions and magnitudes of stars brighter than $V=16$ and declinations between $-30^{\circ}$ and $0^{\circ}$ is presented. The observations have been carried out with the Círculo Meridiano Automático de San Fernando at the Carlos Ulrrico Cesco Observatory in San Juan (Argentina) in the framework of an agreement between the ROA and the OAFA. The final goal of this collaboration is to publish a survey of positions and magnitudes of stars brighter than $V=16$ and with declinations between $-55^{\circ}$ and $+30^{\circ}$.
\end{abstract}

Keywords. astrometry, surveys, catalogs, stars: kinematics

\section{Introduction}

The Círculo Meridiano Automático de San Fernando (CMASF) is a fully automated meridian circle of the Real Instituto y Observatorio de la Armada (ROA) observing since 1996 at the Carlos Ulrrico Cesco Observatory (CUC) in San Juan (Argentina). In December 1999, a CCD camera with $1552 \times 1032$ pixels of 9 microns in size was installed on the CMASF. The main observing program for this instrument is a sky survey within the declination zone $-55^{\circ}<\delta<+30^{\circ}$ and the magnitude range $8<V<16$. The expected positional precision is $\sim 50$ mas in both coordinates and 0.04 mag in $V$. The instrument is managed jointly by the ROA and the Observatorio Astronómico Félix Aguilar (OAFA) of the University of San Juan (Argentina), also owner of the CUC.

In order to test the performance of CMASF, a subcatalogue of positions named the Hispano-Agentinian Meridian Catalogue No. 2 (HAMC2) has been derived for a smaller declination zone $-30^{\circ}<\delta<0^{\circ}$. We expect to publish this subcatalogue in early 2008. In this paper we present preliminary analysis of the subcatalogue and provide comparisons with the UCAC2 and Tycho-2 catalogues, that we have made to investigate possible systematic errors.

\section{Observations}

The CMASF observations reported here have been carried out from December 1999 to May 2006. This automated meridian circle has a $176 \mathrm{~mm}$ aperture and a $2660 \mathrm{~mm}$ focal length (0.'69/pixel). It is located at the CUC observatory on the eastern slopes of Andes with the following geographic coordinates: longitude $69^{\circ} \mathrm{W}$, latitude of $31^{\circ} \mathrm{S}$, altitude $2330 \mathrm{~m}$ above sea level.

The instrument operates in the drift-scan mode (Stone 1993) by observing each night several strips of the sky with a width of $18^{\prime}$ in declination each but having a variable length in right ascension $\left(20^{\mathrm{m}}<\mathrm{RA}<3^{\mathrm{h}}\right)$. Then, a zone to be observed is divided into the 
sub-bands separated by $10^{\prime}$ in declination, so that each sub-band has an $8^{\prime}$ overlap in declination between the adjacent strips (see Evans, Irvin \& Helmer 2002).

\section{Reductions of observations}

Initial reductions include detection of stellar images, their centering and deriving a number of parameters characterizing the images in each strip. Then, the Tycho- 2 catalogue (Høg et al. 2000) is used as a reference frame to obtain preliminary ICRS coordinates in each strip at the epoch of observations. As soon as the observations in a zone of the sky are completed, the final reductions are carried out. For each strip, atmospheric fluctuations of the night are removed using a calibration function based on the overlapping bands between the current strip and the five adjacent strips above and another five adjacent strips below it (Evans, Irwin \& Helmer 2002). Then a catalogue is formed by averaging right ascensions, declinations, and magnitudes of the stars. A more complete description of the method used to form the HAMC2 can be found in the documentation of the Carlsberg Meridian Catalogue 14 (2005).

\section{Comparison with other catalogues}

To investigate possible systematic errors, comparisons have been made between the HAMC2 positions and magnitudes and those in the UCAC2 (Zacharias et al. 2004) and Tycho-2 (Høg et al. 2000) to investigates possible systematic errors. No such error were found. The best achieved average formal precision of HAMC2 positions is $30-40$ mas at $V \sim 12$.

\section{Conclusions}

The HAMC2 subcatalogue presented here shows that the precision in positions reaches the expectations for this survey. No systematic errors have been detected.

\section{Acknowledgements}

We would like to acknowledge Dafydd W. Evans who kindly provided software to construct the catalogue. Also, our gratitude is extended to the Estado Mayor de la Armada and the Ministerio de Educación y Ciencias (AYA2005-24974-E) of Spain and to the San Juan University of Argentina for financial support of this project.

\section{References}

Carlsberg Meridian Catalogue, La Palma 2005, No. 14, Copenhagen University Observatory, Institute of Astronomy of Cambridge, Real Instituto y Observatorio de la Armada en San Fernando

Evans D., Irwin M., \& Helmer L. 2002, A\& A, 395, 347

Høg E., Fabricius C., Makarov V. V., Urban S., Corbin T., Wycoff G., Bastian U., Schwekendiek, \& Wicenec A. 2000, A\&A, 355, L27

Stone R. C. 1993, in Developments in Astrometry and Their Impact on Astrophysics and Geodynamics, Kluwer Academic Publishers, p. 65

Zacharias N., Urban S. E., Zacharias M. I., Wycoff G. L., Hall D. M., Monet D. G., \& Rafferty T. J. 2004, AJ, 127, 3043 